\title{
The Strange Shapes of Cooling Droplets
}

\section{Researchers uncover the mechanism that makes some oil droplets change shape from spheres to icosahedrons to flattened plates.}

\author{
By Erika K. Carlson
}

$\square$ trange things occur when a droplet made of an alkane oil and surfactants is placed in water and cooled. The alkane and surfactant molecules form a solid shell, separating the oil from the surrounding water. Then, as the temperature drops, the shape of this shell changes from a sphere to a 20-sided icosahedron to a flattened hexagonal plate. Now, Ireth García-Aguilar of Leiden University in the Netherlands and colleagues have developed a detailed model to explain these transformations [1].

Previously, other researchers had proposed that the icosahedral forms are caused by some interplay between surface tension and topological defects in the droplet's shell. This mechanism has been observed in some viruses, whose shells can morph from spherical into icosahedral shapes. But this explanation fails for emulsion droplets such as those studied by García-Aguilar and colleagues. For viruses, larger shells tend to be icosahedral, while smaller ones tend to be spherical. For emulsion droplets, the opposite seems to be true: Smaller shells change shape more readily as temperatures

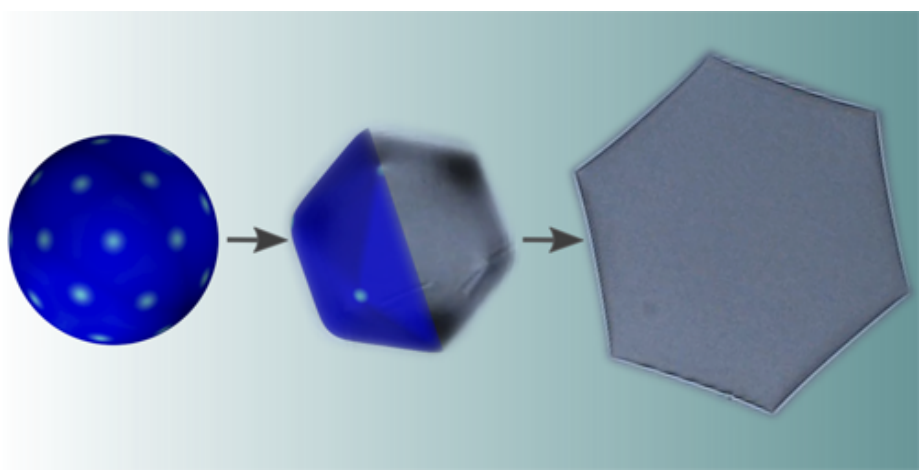

Credit: I. García-Aguilar/Leiden Univ.; E. Sloutskin/Bar-Ilan Univ. drop, while larger shells remain spherical for longer.

In a new model of the phenomenon, García-Aguilar and colleagues included surface tension and defects (as previous models did), but they added two new factors-gravity and a property called spontaneous curvature, which accounts for any preferred curvature possessed by the molecules forming the shell. With these additional ingredients, they found that their model matches observations of emulsion droplets fairly well, including the size-dependence of the shape transitions. Gravity was key in explaining why the droplets, when flattened into plates, always seem to face the same way under a microscope-they flatten in the direction of Earth's gravity, minimizing their energy.

Erika K. Carlson is a Corresponding Editor for Physics based in New York City.

\section{REFERENCES}

1. I. García-Aguilar et al., "Faceting and flattening of emulsion droplets: A mechanical model," Phys. Rev. Lett. 126, 038001 (2021). 